\title{
The global burden of paediatric heart disease
}

\author{
Ndidiamaka L. Musa, ${ }^{1}$ Vibeke Hjortdal, ${ }^{2}$ Bistra Zheleva, ${ }^{3}$ Indah K. Murni, ${ }^{4}$ Shunji Sano, ${ }^{5}$ Steven Schwartz, ${ }^{6}$ \\ Sandra L. Staveski ${ }^{7}$ \\ ${ }^{1}$ Department of Pediatrics, University of Washington, Division of Paediatric Critical Care Medicine, Seattle Children's \\ Hospital Seattle, Seattle, Washington, United States of America; ${ }^{2}$ Department of Cardiothoracic Surgery, Institute of \\ Clinical Research, Aarbus University Hospital, Aarbus, Denmark; ${ }^{3}$ Children's HeartLink, Minneapolis, Minnesota, \\ United States of America; ${ }^{4}$ Department of Pediatrics, Dr Sardjito Hospital/Faculty of Medicine, Universitas Gadjah \\ Mada, Yogyakarta, Indonesia; ${ }^{5}$ Division of Pediatric Cardiothoracic Surgery, University California San Francisco, \\ Paediatric Cardiac Center, Benioff Children's Hospital, San Francisco, California, United States of America; \\ ${ }^{6}$ Departments of Critical Care Medicine and Pediatrics, University of Toronto, The Hospital for Sick Children, Toronto, \\ Ontario, Canada; ${ }^{7}$ Cincinnati Children's Hospital Medical Center, Research in Patient Services, Cincinnati, Ohio, \\ United States of America
}

\begin{abstract}
An estimated 15 million children die or are crippled annually by treatable or preventable heart disease in low- and middle-income countries. Global efforts to reduce under- 5 mortality have focused on reducing death from communicable diseases in low- and middle-income countries with little to no attention focusing on paediatric CHD and acquired heart disease. Lack of awareness of CHD and acquired heart disease, access to care, poor healthcare infrastructure, competing health priorities, and a critical shortage of specialists are important reasons why paediatric heart disease has not been addressed in low resourced settings. Non-governmental organisations have taken the lead to address these challenges. This review describes the global burden of paediatric heart disease and strategies to improve the quality of care for paediatric heart disease. These strategies would improve outcomes for children with heart disease.
\end{abstract}

Keywords: Paediatric heart disease; low- and middle-income countries; humanitarian services

Received: 15 September 2017; Accepted: 15 September 2017

$\mathrm{E}$ FFORTS AT REDUCING MORTALITY AMONG CHILDREN under 5 years of age have been the focus of significant international attention for decades, beginning with the convention on the rights of the child and accelerating in 2000 with the Millennium Development Goals, with goal 4 being to reduce the under-5 mortality rate by two-thirds. This work continued with the United Nations' adoption of the Sustainable Development Goals, specifically Sustainable Development Goal 3, which aims to end preventable deaths of newborns and children under

Correspondence to: N. L. Musa, MD, FCCM, Department of Paediatrics, University of Washington, Division of Paediatric Critical Care Medicine Seattle Children's Hospital, 4800 Sand Point Way NE, FA.2.112, PO Box 5371, Seattle, WA 98145, United States of America. Tel: 206987 2140; Fax: 206-987-3866; E-mail: Ndidi.Musa@seattlechildrens.org
5 years of age. All countries aimed to reduce neonatal mortality to at most 12 per 1000 live births and under-5 mortality to at most 25 per 1000 live births. ${ }^{1,2}$ These efforts have been largely targeted at prevention and treatment of communicable diseases, owing to the number of affected children and the relatively low cost, low technology, and experience required to do this work. Overall, progress has been achieved with a reduction in global mortality. However, it is notable that CHD and acquired heart diseases, which affect a significant number of children, have largely been left to non-governmental organisations to address. This is probably because of perceived challenges around cost, technology, availability of trained personnel, and the expertise required to affect change. $^{3}$ 
In the past 50 years, there have been major breakthroughs in cardiovascular care such as improvements in diagnosis and surgical treatment. Survival of newborns with CHD has increased considerably in high-income countries. Unfortunately, this is not the case in many low- and middle-income countries where the burden is the heaviest and rates of death and disability continue to rise. ${ }^{4}$ Until recently, the global dialogue about the endemic burden of cardiovascular disease among the world's poorest billion people was not addressed, yet globalisation has affected behavioural and metabolic risk factors for cardiovascular disease. It is important to note that the causes of heart disease in high-income countries are different from those in low- and middle-income countries, where ischaemic heart disease is relatively infrequent and hypertensive heart disease, cardiomyopathies, rheumatic heart disease, pericardial disease, and CHD are common. ${ }^{5}$ Additionally, rheumatic heart disease has been virtually eliminated from high-income countries but remains a significant public health issue in low- and middle-income countries and is the leading cause of acquired heart disease. ${ }^{6}$ Thus, paediatric heart disease, both congenital and acquired, remains a major health problem in low- and middle-income countries.

With globalisation and accessibility of echocardiography, the inequalities of access to care are being recognised. Recent history suggests that these barriers are becoming less formidable. Excellent outcomes can be achieved even with low-cost cardiovascular care in challenging environments, India being a leading example. We are learning that specific lesions with excellent long-term prognosis, such as atrial and ventricular septal defects, and tetralogy of Fallot can be managed very well in the vast majority of cases, even when the advanced care and rescue capabilities present in high-income countries are not available.

\section{Incidence and prevalence of paediatric heart disease}

Congenital anomalies are a major cause of infant mortality, and children who survive often live with significant life-long disabilities. About $3 \%$ of all live births are associated with congenital anomalies. ${ }^{8}$ According to the 2010 global burden of disease, injuries, and risk factors study, 302,000 infants died from congenital anomalies, which accounted for $6 \%$ of all infant deaths, with $96 \%$ of these occurring in low- and middle-income countries. ${ }^{9}$ About $28 \%$ of all major congenital anomalies are heart defects. ${ }^{10}$

Most of the data about CHD globally are extrapolated from high-income countries, and quality regional data from low- and middle-income countries are lacking. Adequate documentation of births and mortality is essential to drive a policy shift that would affect access to care for children with heart disease and to fight the perception that CHD rates in low- and middle-income countries are lower than in highincome countries. CHD has an estimated prevalence of 10-12 per 1000 live births and represents $\sim 1.35$ million live births each year. ${ }^{11}$ Reported figures vary worldwide, and a meta-analysis by van der Linde found that the highest CHD birth prevalence was in Asia, 9.3 per 1000 live births, and the lowest in Africa, 1.9 per 1000 live births. ${ }^{10}$ The prevalence of CHD in low- and middle-income countries, especially in Africa, is underestimated owing to a paucity of data, lack of prioritisation of cardiac care services, access to care in general, poor health infrastructure, lack of trained personnel, limited resources, and early mortality.

To the extent that there are reliable studies, they corroborate that rates are similar across the world. A study of 20,307 newborns in India found an overall CHD birth prevalence rate similar to that reported worldwide, at 8.07/1000 live births (95\% CI 6.94-9.4). ${ }^{12}$ Another study from China showed similar results, with 686 having CHD in a cohort of 84,062 births, resulting in an overall incidence of 8.2/1000 total births. ${ }^{13}$ To the best of our knowledge, rates of CHD in low- and middleincome countries are similar to high-income countries and may be higher.

With regard to outcomes, about half of all deaths among children with CHD occur during the first year of life, especially in countries where access to surgery is limited. ${ }^{14}$ In addition, children with CHD that present late frequently suffer from poor nutrition, pulmonary congestion, and pulmonary hypertension, which in turn predisposes these children to pneumonia and sepsis, all of which is challenging to manage in settings with limited resources.

Rheumatic heart disease remains a global health problem in low- and middle-income countries. According to the benchmark estimates of the burden of disease, there are 15.6 million prevalent cases, 282,000 incident cases, and 233,000 deaths attributable to rheumatic fever or rheumatic heart disease annually. ${ }^{15}$ Recent studies in school children have reported the prevalence as high as 21.5-30.4 cases per $1000{ }^{16-18}$ Furthermore, the disability-adjusted life year burden was estimated at 1430 (range 944-2067) in 2010. ${ }^{15}$ These are conservative estimates and the true burden of disease is thought to be even greater. A recent study described mortality rates that were higher than those of rotavirus, meningitis, hepatitis B, and half of those with malaria. ${ }^{19}$ The true burden is probably underestimated because of methodological differences between studies and lack of echocardiographic detection of asymptomatic disease. ${ }^{20}$ 
Additionally, there was a lack of published data from low- and middle-income countries where the burden of disease is the highest. ${ }^{21}$ Previously published studies on prevalence or incidence of rheumatic heart disease were conducted mostly in urban areas, although the prevalence of rheumatic heart disease may be higher in rural areas.

The significance of potentially fatal sequelae of rheumatic heart disease remains under-explored. A previously published study involving 12 African countries, India, and Yemen showed a high prevalence of major cardiovascular complications related to moderate-to-severe multivalvular disease including congestive heart failure, pulmonary hypertension, atrial fibrillation, stroke, infective endocarditis, and major bleeding. ${ }^{22}$ In addition, a systematic review consisting of 16 studies in South Africa revealed that the overall 60-day mortality after admission with acute heart failure due to rheumatic heart disease was 24.8\% (95\% CI, 13.6-42.5\%), and 180-day mortality was $35.4 \%$ (95\% CI, 21.6-54.4\%). ${ }^{23}$ Zühlke et al found, in 3343 children and adults from 14 low- and middle-income countries, that the 2-year case fatality rate was $16.9 \%$, median age at death was 28.7 years, and complications of congestive heart failure and stroke affected one-fifth of patients over 2 years of age. ${ }^{24}$ Thus, programmes focusing on early detection and treatment of clinical rheumatic heart disease are required to improve outcomes.

Secondary prophylaxis has been proven to be costeffective in controlling rheumatic heart disease as repeated streptococcal infections are thought to prime a susceptible individual and result in a heightened response to an infection from rheumatogenic streptococcus, which in turn drives the valvular inflammatory response. However, most low- and middle-income countries still do not have effective secondary prophylaxis programmes. Furthermore, rheumatic heart disease can go undetected and result in patients presenting with debilitating heart failure. At this stage, surgery is the only possible treatment option. In low- and middle-income countries, limitations of human resources and facilities for cardiac surgery and post-operative management may adversely affect the outcomes of the paediatric cardiac surgery for these children. Finally, providing anticoagulation services in low- and middle-income countries after surgery is problematic.

\section{Addressing the global burden}

There are important considerations in addressing this burden. The first step is recognising the contributing factors. The care of children with CHD and rheumatic heart disease in low- and middle-income countries settings is challenging. Most CHD are not diagnosed before birth owing to lack of antenatal screening. Early diagnosis of simple lesions could result in early referral and treatment before the onset of irreversible sequelae. Other factors that contribute include limitations in the existing infrastructure, lack of resources, low numbers of appropriately trained healthcare workers, and a general lack of awareness of heart disease in children. Meeting these challenges requires clinical evidence pertinent to the local settings, an adequate number of well-trained personnel, improvement in nursing and medical education, research, and quality improvement activities. In addition, there is increasing realisation that the lack of facilities for sustainable paediatric cardiac services in low- and middle-income countries results in a massive number of preventable deaths and ongoing suffering. It is estimated that 15 million children die or are crippled annually by potentially treatable or preventable cardiac disease. ${ }^{25}$ Paediatric cardiovascular services are highly specialised and require significant financial resources. In high-income countries where the national needs of paediatric cardiac surgery are met, around 450 interventional treatments or surgical procedures for $\mathrm{CHD}$ are done yearly per 5 million inhabitants. ${ }^{26}$ In low- and middle-income countries, with 3-4 times the birth rate and with the additional need for surgical treatment for rheumatic heart diseases, the need for cardiovascular services is much greater. ${ }^{13}$ The work led by nongovernmental organisations has shown that these barriers in training and organisation can be overcome, but it will take a very long time to do this with current models.

\section{Access to cardiac care}

In the poorest parts of Africa, Asia, and South and Central America, there are limited paediatric cardiovascular services. Some of the larger cities have facilities for diagnosis and intervention and operation of selected and straightforward conditions in children beyond infancy. Infant and newborn open heart surgery is limited, if present at all. Requirements for developing a sustainable and comprehensive paediatric cardiovascular service are demanding and need significant resources. The treatment of the four most common CHD - atrial and ventricular septal defects, patent ductus arteriosus, and tetralogy of Fallot covers approximately half of the need. The children can be treated on an elective basis, transported over long distances to a facility, and the heart disease is likely resolved, at least for several years or even decades, with one operation or intervention. Open heart surgery and catheter interventions of newborns and infants require a major increase in resources, infrastructure, and expertise among physicians, nurses, paramedics, and families. 


\section{Expenditure}

Health expenditure is a large part of the gross domestic product in high-income countries compared with the tiny fraction it is in most low-income countries. There is great disparity in access to paediatric cardiovascular services worldwide. Many countries struggle to meet basic primary health needs, therefore making it impossible to provide tertiary paediatric cardiovascular services. ${ }^{27}$ There are strategies such as developing partnerships through non-governmental organisations and governments and humanitarian outreach that are helping to defray costs while building capacity and sustainable programmes. In total, $58 \%$ of the burden of disease of CHD could be averted by scaling up selected surgical care in low- and middle-income countries through various mechanisms. ${ }^{28}$ The experience of highincome countries has shown that, as economies grow and health systems strengthen, childhood mortality from preventable diseases starts to decline, and CHD surfaces as one of the top contributors to neonatal and under- 5 mortality. The World Bank predicts that the world is very close to achieving the 2030 goal of eliminating poverty, and it is likely that many countries will emerge with a significant and growing burden of childhood heart disease. ${ }^{29}$

\section{Building buman capacity}

Sub-Saharan Africa carries over $24 \%$ of the global disease burden but is home to only $3 \%$ of the global health workforce, in sharp contrast to North America with only $10 \%$ of the world disease burden but $37 \%$ of the global health workforce. ${ }^{30}$ This inequity represents one of the greatest impediments to healthcare in Sub-Saharan Africa. In addition, Africa has been plagued by a high attrition rate of qualified staff and significant migration of healthcare professionals, either to richer nations or to local private organisations for higher remuneration, better career opportunities, and improved working environment. There is a great need for healthcare workers to be trained, sustained, and retained in Sub-Saharan Africa and other low- and middle-income countries. $^{6}$ The international strategies to provide help to children with CHD in low- and middle-income countries come mainly from non-governmental organisations. Voluntary and humanitarian initiatives have centred around transporting children with heart disease to high-income countries, treating children during surgical missions, and long-term humanitarian training exchanges that help to develop sustainable cardiovascular centres. The real benefits from the first two strategies are variable as only a small number of children can be served, they are associated with high costs, and may not be sustainable. In addition, these efforts have been criticised as a form of medical tourism. Kingham et al described the number and cost of short-term medical missions and training exchanges from the United States to low- and middle-income countries at 6000, with an associated cost of United States $\$ 250$ million, but with no exact figures on the quality and quantity of these sessions. ${ }^{31}$ Currently, non-governmental organisations recognise the need to build sustainable programmes locally through training and empowerment of the local team, rather than saving individual children by bringing them to institutions in high-income countries.

Building cardiovascular centres through a sustained strong international partnership is a key strategy to build, strengthen, and maintain competence of healthcare workers. ${ }^{27}$ The paediatric cardiac unit in Guatemala City is an excellent example of a sustainable programme that was established through a joint venture between the Aldo Castaneda Foundation, the government of Guatemala, and donations from abroad that have trained cardiac surgeons in the country and region. ${ }^{18}$ Another example is the partnership between Children's National Health System (Washington, DC) and Samaritan's Purse Children's Heart Project, which developed the Ugandan Heart Institute. ${ }^{32}$ Dearani et al outlined principles for building successful partnerships in low- and middleincome countries as "a commitment to work together in the spirit of collaboration, to track and measure results, to establish and designate an acceptable structure and to build local government and community support."33

\section{Building infrastructure}

Educating government and community leaders about prevention and treatment of paediatric heart disease is imperative because building sustainable cardiovascular services is complex and expensive. ${ }^{25}$ Building high-quality cardiovascular services in low- and middle-income countries requires intensive coordinated efforts, infrastructure development, identification of key stakeholders, and building collegial relationships for ongoing consultation and mentorship. Infrastructure development requires a significant amount of capital, building new or renovating old facilities, purchasing equipment, support services such as pharmacy, blood bank, operating rooms, and intensive care resources, and, most importantly, human resources. ${ }^{25}$

\section{Research and quality improvement}

Encouraging quality improvement and research to define the scope of the problem, resources available, 
and best strategies are needed. Reddy et al published a report on the effect of preoperative determinants on early outcomes of 1028 consecutive infant heart operations in India. ${ }^{34}$ The International Quality Improvement Collaborative for Congenital Heart Surgery in Developing World Countries is an initiative allowing paediatric cardiovascular centres from low- and middle-income countries to work together to lower congenital heart surgery mortality. To date, 58 sites from 24 countries have entered 60,607 cases in the International Quality Improvement Collaborative for Congenital Heart Surgery in Developing World Countries patient registry. Teams also benefit from free participation in monthly educational webinars from Boston Children's Hospital on topics such as team-based practice, infection prevention, and safe perioperative practices. Current International Quality Improvement Collaborative for Congenital Heart Surgery in Developing World Countries data show that participating hospitals were able to lower infection rates and mortality. ${ }^{35}$

\section{Conclusion}

In its advocacy series, The Invisible Child, Children's HeartLink highlighted several important recommendations that multilateral and financing agencies, the World Health Organization, national and local governments, research and teaching institutions, non-governmental organisations, patient and family advocates, and the private sector need to undertake to ensure that children around the world have access to quality and sustainable paediatric care close to where they live. The recommendations focus on the following:

- Investments in increasing capacity at all levels of the healthcare delivery system to screen, diagnose, and treat children with heart disease,

- Building accredited paediatric cardiac training programmes in all regions globally to assure systematic recognition of the basic signs and symptoms of CHD and rheumatic heart disease,

- Improve surveillance through systematic data collection on paediatric heart disease in national health surveys and include burden of disease and cause of child death statistics, and

- Ensuring that paediatric cardiac care will be included in benefits packages in universal health coverage and social protection platforms, and protecting patients from unaffordable expenses related to their care. ${ }^{36}$

We think that is it appropriate for global health agencies to consider CHD and acquired heart disease as targets for improving access to care worldwide, given the great potential for life-time benefit.

\section{Acknowledgements}

None.

\section{Financial Support}

This paper received no specific grant from any funding agency, commercial, or not-for-profit sectors.

\section{Conflicts of Interest}

None.

\section{References}

1. UNDP Data. Retrieved May 17, 2017, from http://www.undp.org/ content/undp/en/home/sustainable-development-goals/goal-3-goodhealth-and-well-being/targets.

2. Forouzanfar MH, Afshin A, Alexander LT, et al. Global, regional, and national comparative risk assessment of 79 behavioral, environmental and occupational, and metabolic risks or clusters of risks, 1990-2015: a systematic analysis for the Global Burden of Disease Study 2015. Lancet 2016; 388: 1659-1724.

3. Penny DJ. Global perspectives on paediatric cardiac critical care. Pediatr Crit Care Med 2016; 17: S388-S393.

4. Zheleva B, Atwood JB. The invisible child: childhood heart disease in global health. Lancet 2017; 389: 16-18.

5. Kwan GF, Mayosi BM, Mocumbi AO, et al. Endemic cardiovascular diseases of the poorest billion. Circulation 2016; 133: 2561-2575.

6. Zühlke L, Mirabel M, Marijon E. Congenital heart disease and rheumatic heart disease in Africa: recent advances and current priorities. Heart 2013; 99: 1554-1561.

7. Nguyen N, Leon-Wyss J, Iyer KS, Pezzella AT. Paediatric cardiac surgery in low-income and middle-income countries: a continuing challenge. Arch Dis Child 2015; 100: 1156-1159.

8. Higashi H, Barendregt JJ, Vos T. The burden of congenital anomalies amenable to surgeries in low-income and middle-income countries: a modeled analysis. Lancet 2013; 381: S62.

9. Murray CJL, Vos T, Lozano R, et al. Disability-adjusted life years (DALYs) for 291 diseases and injuries in 21 regions, 1990-2010: a systematic analysis for the Global Burden of Disease Study 2010. Lancet 2012; 380: 2197-2223.

10. van der Linde D, Konings EEM, Slager MA, et al. Birth prevalence of congenital heart disease worldwide. J Am Coll Cardiol 2011; 58: 2241-2247.

11. Hoffman JIE. The global burden of congenital heart disease: a review article. Cardiovasc J Afr 2013; 24: 141-145.

12. Saxena A, Mehta A, Sharma M, et al. Birth prevalence of congenital heart disease: a cross-sectional observational study from North India. Ann Pediatr Cardiol 2016; 9: 205-206.

13. Qu Y, Liu X, Zhuang J, et al. Incidence of congenital heart disease: the 9-year experience of the Guangdong Registry of Congenital Heart Disease, China. PLoS ONE 2016; 11: 7-12.

14. Hewitson J, Zilla P. Children's heart disease in sub-Saharan Africa: challenging the burden of disease. SA Heart 2010; 7: 18-29.

15. Wyber R, Zühlke L, Carapetis J. The case for global investment in rheumatic heart-disease control. Bull World Health Organ 2014; 92: 768-770.

16. Marijon E, Tivane A, Voicu S, et al. Prevalence of congenital heart disease in schoolchildren of sub-Saharan Africa, Mozambique. Int J Cardiol 2006; 113: 440-441.

17. Carapetis JR, Zühlke L, Taubert K, Narula J. Continued Challenge of Rheumatic Heart Disease. Glob Heart 2013; 8: 185-186.

18. Saxena A. Strategies for the improvement of cardiac care services in developing countries: what does the future hold. Future Cardiol 2017; 8: 29-38 
19. David W. Rheumatic fever: neglected again. Science 2009; 324: $1-3$.

20. Zühlke LJ, Steer AC. Estimates of the global burden of rheumatic heart disease. Glob Heart 2013; 8: 189-195.

21. Jackson SJ, Steer AC, Campbell H. Systematic review: estimation of the global burden of non-suppurative sequelae of upper respiratory tract infection: rheumatic fever and post-streptococcal glomerulonephritis. Trop Med Int Health 2010; 16: 2-11.

22. Zuhlke L, Engel ME, Karthikeyan G, et al. Characteristics, complications, and gaps in evidence-based interventions in rheumatic heart disease: The Global Rheumatic Heart Disease Registry (the REMEDY study). Eur Heart J 2015; 36: 1115-1122.

23. Zühlke LJ, Engel ME, Watkins D, Mayosi BM. Incidence, prevalence, and outcome of rheumatic heart disease in South Africa: a systematic review of contemporary studies. Int J Cardiol 2015; 199: 375-383.

24. Zühlke L, Karthikeyan G, Engel ME, et al. Clinical outcomes in 3343 children and adults with rheumatic heart disease from 14 low- and middle-income countries two-year follow-up of the global rheumatic heart disease registry (the REMEDY Study). Circulation 2016; 134: 1456-1466.

25. Yacoub MH. Establishing paediatric cardiovascular services in the developing world: a wake-up call. Circulation 2007; 116: 1876-1878.

26. Larsen $\mathrm{S}$, Olsen $\mathrm{M}$, Emmertsen $\mathrm{K}$, et al. Interventional treatment of patients with congenital heart disease. J Am Coll Cardiol 2017; 26: 2752-2732.

27. Leblanc JG. Creating a global climate for paediatric cardiac care. World J Pediatr 2009; 5: 89-92.
28. Debas H, Donkor P, Gawande A, Jamison D, Kruk M, Mock C. Disease Control Priorities, 3rd edn, Vol 1. In Essential Surgery. World Bank, Washington, DC, 2015: 32.

29. The World Bank. Global monitoring report 2014/2015: ending poverty and sharing prosperity. The World Bank [Serial online] 2014. Retrieved May 14, 2017, from, https://openknowledge. worldbank.org/handle/ 10986/20330.

30. Anyangwe S, Mtonga C. Inequities in the global health workforce: the greatest impediment to health in Sub-Saharan Africa. Int J Environ Res Public Health 2007; 4: 93-100.

31. Kingham TP, Price RR, Casey KM, Rogers SO, Kushner AI. Beyond volunteerism: augmenting surgical care in resource-limited settings. Bull Am Coll Surg 2011; 96: 16-21.

32. Aliku T, Lubega S, Namuyonga J, et al. Paediatric cardiovascular care in Uganda: current status, challenges, and opportunities for the future. Ann Pediatr Card 2017; 10: 50-59.

33. Dearani JA, Neirotti R, Kohnke EJ, et al. Improving Paediatric cardiac surgical care in developing countries: matching resources to needs. Semin Thorac Cardiovasc Surg Pediatr Card Surg Ann 2010; 13: 35-43.

34. Reddy NS, Kappanayil M, Balachandran R, et al. Preoperative determinants of outcomes of infant heart surgery in a limitedresource setting. Semin Thorac Cardiovasc Surg 2015; 27: 331-338.

35. Jenkins KJ, Castañeda AR, Cherian KM, et al. Reducing mortality and infections after congenital heart surgery in the developing world. Paediatrics 2014; 134: e1422-e1430.

36. A voice for the invisible child: childhood heart disease and the global agenda. [Serial online], 2016. Retrieved May 13, 2017, from, http://theinvisiblechild.childrensheartlink.org/The-InvisibleChild-Brief-Four.pdf. 